\title{
FITOTERAPIA, SUSTENTABILIDADE E SAÚDE PÚBLICA: EFEITOS DA CAMELLIA SINENSIS NA PERDA DE PESO
}

\author{
Tiago Ferrari ${ }^{1}$, Vinicius Maia ${ }^{2}$, Daniele Fernanda Felipe ${ }^{3}$, Ariana Ferrari $^{3}$ \\ ${ }^{1}$ Médico do Departamento de Medicina Intensiva, Hospital Municipal de Maringá, \\ Maringá, Maringá-PR, Brasil \\ ${ }^{2}$ Acadêmico do Curso de Medicina da Universidade Cesumar - UNICESUMAR, \\ Maringá-PR, Brasil \\ ${ }^{3}$ Docente do Programa de Pós-graduação em Tecnologias Limpas da Universidade \\ Cesumar - UNICESUMAR, Maringá-PR, Brasil \\ E-mail: ariana.ferrari@unicesumar.edu.br
}

\author{
Recebido em: 15/11/2021 - Aprovado em: 15/12/2021 - Publicado em: 30/12/2021 \\ DOI: 10.18677/EnciBio 2021D26 \\ trabalho licenciado sob licença Creative Commons Attribution-NonCommercial-NoDerivatives 4.0 International License
}

\begin{abstract}
RESUMO
A prevalência da obesidade teve um aumento exponencial, sendo considerado um grave problema de saúde pública no mundo, levando ao crescente interesse pelo tratamento terapêutico. Na fitoterapia um dos alimentos que vem se destacando devido ao seu potencial antioxidante e anti-inflamatório é o chá verde. O objetivo desse trabalho foi realizar uma revisão bibliográfica a respeito da capacidade funcional do chá verde na perda de peso. Foram analisados estudos em livros e artigos científicos, tendo como critério de inclusão as palavras obesidade, alimentos funcionais, chá verde ou Camellia Sinensis como título, resumo ou palavra-chave e como exclusão a ausência desses termos. O chá verde é um fitoterápico a ser utilizado na saúde pública por ser uma alternativa viável, acessível, segura e de baixo custo. Sua ação na perda de peso ocorre principalmente por atuar como um potente antioxidante além de exercer ações termogênicas. Assim, o chá verde pode auxiliar na prevenção e tratamento da obesidade, porém, ainda são necessários mais estudos para definir a dose diária de chá verde recomendada.
\end{abstract}

PALAVRAS-CHAVE: chá verde, obesidade, saúde pública.

\section{PHYTOTHERAPY, SUSTAINABILITY AND PUBLIC HEALTH: EFFECTS OF CAMELLIA SINENSIS ON WEIGHT LOSS}

\begin{abstract}
The prevalence of obesity has increased exponentially, being considered a serious public health problem worldwide, leading to growing interest in therapeutic treatment. In herbal medicine, one of the foods that has stood out due to its antioxidant and antiinflammatory potential is green tea. The objective of this work was to carry out a literature review regarding the functional capacity of green tea in weight loss. Studies in books and scientific articles were analyzed, having as inclusion criteria the words obesity, functional foods, green tea or Camellia Sinensis as title, abstract or keyword, and the absence of these terms as exclusion. Green tea is an herbal medicine to be ENCICLOPÉDIA BIOSFERA, Centro Científico Conhecer - Jandaia-GO, v.18 n.38; p. 344 2021
\end{abstract}


used in public health as it is a viable, accessible, safe and low-cost alternative. Its action in weight loss occurs mainly by acting as a powerful antioxidant in addition to exerting thermogenic actions. Thus, green tea can help prevent and treat obesity, however, further studies are needed to define the recommended daily dose of green tea.

KEYWORDS: green tea, obesity, public health.

\section{INTRODUÇÃO}

A obesidade é uma doença crônica não transmissível (DCNT), caracterizada por um acúmulo excessivo de gordura corporal, a ponto de comprometer a saúde física e psicológica do indivíduo e reduzir a expectativa de vida (SARMA et al., 2021). Dentre os fatores de risco pode-se citar o elevado consumo de alimentos com alta densidade calórica, ricos em gordura saturada e açúcar, e uma diminuição no consumo de carboidratos complexos, frutas e legumes, bem como o sedentarismo (SCHWARTZ et al., 2017).

A obesidade é considerada um dos maiores problemas de saúde pública no mundo. Para o ano de 2025 estima-se que 18\% dos homens e $21 \%$ das mulheres serão obesas (BARAKAT; ALMEIDA, 2021). De acordo com dados do Vigitel uma pesquisa que investiga as DCNT através de inquéritos telefônicos, o Brasil apresenta um percentual de $19,8 \%$ de adultos obesos, contudo, observa-se uma prevalência ligeiramente maior em mulheres, com 20,7\%, do que nos homens $18,7 \%$. Ainda conforme a pesquisa, quando se trata de excesso de peso esse percentual é acima do dobro, com uma frequência de $55,7 \%$, porém ao contrário da obesidade, o sobrepeso é maior entre os homens $(57,8 \%)$ quando comparado com as mulheres (53,9\%) (BRASIL, 2019).

Em virtude do aumento da obesidade e sua correlação com várias doenças, uma grande parcela da população, tem se direcionado em busca de alternativas para uma alimentação saudável e balanceada, através de uma rotina alimentar composta por alimentos saudáveis e funcionais, bem como o interesse pelo uso de produtos e suplementos que auxiliam na redução de peso, prevenção e no tratamento da obesidade (OHISHI et al., 2021).

A OMS considera a utilização da fitoterapia valiosa na assistência farmacêutica. Com base nisso, a Política Nacional de Práticas Integrativas e Complementares no SUS e a Política Nacional de Plantas Medicinais e Fitoterápicos no Brasil foram implementadas no intuito de fortalecer a utilização das plantas medicinais na saúde pública, elaborando ações e programas que envolvam o uso dessa prática (BRASIL, 2006). O chá verde é rico em polifenóis, obtido através das folhas frescas da erva Camellia Sinensis, e seus subprodutos, sendo capazes de promover a diminuição de peso corporal, gordura corporal e auxiliar na prevenção e tratamento da obesidade e de doenças associadas como diabetes, doenças cardiovasculares e dislipidemias (GHOSH et al., 2021).

A pesquisa fundamentou-se em um estudo de revisão narrativa da literatura, no qual foram realizadas buscas de dados on-line PUBMED, LILACS e SCIELO, preferencialmente dos últimos cinco anos. Os descritores utilizados na busca foram: fitoterapia, saúde pública, sustentabilidade, chá verde, Camellia Sinensis, oxidação lipídica, características funcionais, obesidade. Foram estabelecidos como critérios de inclusão os artigos publicados em português, espanhol e inglês, artigos que retratassem a temática referente às características funcionais do chá verde. Os 
artigos foram selecionados aleatoriamente, na medida em que apareciam na busca eletrônica, através das palavras-chave.

Desse modo, o presente artigo teve como objetivo fazer uma revisão bibliográfica a respeito da capacidade funcional do chá verde na perda de peso, com enfoque da sua utilização na saúde pública.

\section{FITOTERAPIA, SUSTENTABILIDADE E SAÚDE PÚBLICA}

A aplicabilidade das plantas medicinais descende de uma prática milenar, cuja disposição encontra-se na prevenção, bem como no tratamento de doenças, presentes nas diferentes culturas em todo o mundo e relacionadas aos primórdios da medicina (ALFERINK et al., 2017; POSWAL et al., 2019). O uso de plantas medicinais originou o conceito de fitoterapia e tem se tornado alvo de pesquisas científicas, já que a aplicação está pautada em comprovação perante as áreas de estudos dos fotoquímicos e da farmacologia (FALZON; BALABANOVA, 2017). Além disso, em países desenvolvidos a fitoterapia é uma alternativa de tratamento mais saudável ou com menos efeitos adversos (MATTOS et al., 2018).

O Brasil tem um histórico de uso das plantas medicinais através do consumo alimentar, como medicamentos no tratamento de várias doenças ou na aplicação de cosméticos (JHEMES et al., 2017). Esse cenário já era observado pelas antigas civilizações, como romanos, hebreus, chineses, indianos, árabes, gregos e pelos egípcios (SANTOS; TRINDADE, 2017; SANTANA et al., 2018; GOULARTE et al., 2021).

É importante salientar que o Brasil tem um grande potencial para o desenvolvimento dessa prática terapêutica, já que possui uma vasta diversidade de espécies vegetais, uma ampla sócio diversidade, com o uso de plantas medicinais vinculado ao conhecimento tradicional, e ainda, a tecnologia para validar cientificamente este conhecimento (BRASIL, 2006). O País abriga cerca de $14 \%$ da diversidade de plantas do mundo, o que equivale a cerca de 45,3 a 49,5 mil o número de espécies de plantas (PEIXOTO; MORIM, 2003). Existem ainda cerca de mais de 350 mil espécies, as quais apresentam um total de 1,8 milhão de espécies biológicas (JHEMES et al., 2017). Além disso, avalia-se que pelo menos metade das plantas contenham substâncias chamadas de princípios ativos, as quais têm propriedades curativas e preventivas para muitas doenças (PELTZER; PENGPID, 2019).

O Brasil ainda é visto em destaque por possuir um terço da flora mundial, além de ser a Amazônia a maior reserva de produtos naturais com ação fitoterápica do planeta (BRASIL, 2006). Sendo que se exploradas de maneira sustentável, a terapia com plantas gera renda, além de contemplar a preservação da natureza e das espécies. Ressalta-se ainda, sobre a importância dos estudos etno botânicos, já que, estes possibilitam o resgate e a preservação dos conhecimentos populares das comunidades envolvidas (ALFERINK et al., 2017). Verifica-se ainda que na Constituição Brasileira, no artigo 225, é assegurado o direito de todos a terem 0 meio ambiente ecologicamente equilibrado, e incumbe ao poder público e à coletividade o dever de proteger, preservar, defender e disseminar ações e bons hábitos que contribuam para a melhoria na qualidade de vida, para a atual e as futuras gerações (BRASIL, 2016).

A partir da década de 1990, o uso das Práticas Integrativas e Complementares (PIC) tem aumentado em proporções mundiais. Nesse ínterim, o 
Brasil, com o intuito de encorajar a implementação do uso das práticas nãoconvencionais em saúde (PNCS), estabeleceu diretrizes na área de plantas medicinais e saúde pública no ano de 2006, com a publicação da Política Nacional de Práticas Integrativas e Complementares (PNPIC), com viés político, técnico, econômico, social e cultural, dirigida aos usuários do Sistema Único de Saúde, principalmente no âmbito da Atenção Primária à Saúde (APS) (RUELA et al., 2019). Isso ocorreu a partir da Portaria do ministério da saúde no 971, de 03 de maio de 2006, a qual entre outras práticas integrativas está a fitoterapia, adotada como um recurso terapêutico, caracterizado pelo uso de plantas medicinais em diferentes formas farmacêuticas (MATTOS et al., 2018).

Além disso, no mesmo ano criou-se por meio do Decreto ํㅜ 5.813, de 22 de junho de 2006, a Política Nacional de Plantas Medicinais e Fitoterápicos (PNPMF), com o objetivo de implementar ações para promoção da qualidade de vida da população brasileira através do acesso seguro e racional da fitoterapia no cotidiano das pessoas. Além disso, a PNPMF tem como meta o desenvolvimento de tecnologias e inovações, fortalecimento das cadeias e dos arranjos produtivos, uso sustentável da biodiversidade brasileira e o desenvolvimento do complexo produtivo da saúde (BRASIL, 2006). Em relação a sustentabilidade, sabe-se que o Brasil detém a maior parcela da biodiversidade mundial, em torno de 15 a $20 \%$. Entre os anos de 2004 a 2014 houve um aumento de três vezes no que tange aos municípios que ofertavam fitoterapia na APS (MATTOS et al., 2018), sendo que, as plantas medicinais e fitoterapia se destacam entre as demais ofertadas, representando $30 \%$ dos municípios (TESSER et al., 2018).

A adoção desses sistemas integracionais permite a estimulação dos mecanismos naturais de prevenção e recuperação da saúde, através de tecnologias eficazes e seguras, facilitando a integração do ser humano com o meio ambiente e a sociedade (MIRANDA; UHLMANN, 2021). São denominadas complementares, porque assim como a medicina tradicional ocidental baseada em medicamentos sintéticos, a complementar deve ter o seu espaço na assistência médica, visto que ambas têm por objetivo favorecer a saúde e, assim, podem ser usadas em conjunto (DACAL; SILVA, 2018).

Uma das metas a serem atingidas pela ONU, até o ano de 2030, através da agenda com os "Objetivos de Desenvolvimento Sustentável", é assegurar vida saudável e promover o bem-estar para todos, garantir o acesso a medicamentos seguros, eficazes, de qualidade e a preços acessíveis para todos, de forma que a fitoterapia se enquadra neste padrão almejado como uma alternativa. Nota-se ainda, que essa abordagem impulsiona o desenvolvimento comunitário, a solidariedade e participação social (HASENCLEVER et al., 2017).

Assim, entende-se que a Educação Ambiental se refere a uma nova perspectiva, que atua na preservação das espécies, uma reaproximação do ser humano com a natureza, bem como, a conservação do conhecimento popular transmitido por meio de gerações (CECCHIN; FERREIRA, 2021). Neste sentido, a fitoterapia promove uma conexão entre os indivíduos e a natureza, por ser uma parte integrante da cultura já desenvolvida e praticada na sociedade a partir da vegetação, na qual buscam-se formas de ajudar o organismo em vários aspectos, como por exemplo, restaurar a imunidade enfraquecida, normalizar funções fisiológicas, desintoxicar órgãos e até mesmo para o rejuvenescimento. Ou seja, a fitoterapia 
permite o contato da população com sua própria história e cultura (MIRANDA; UHLMANN, 2021).

No tocante, a OMS destaca que cerca de $80 \%$ da população dos países em desenvolvimento adotam as práticas tradicionais direcionadas às necessidades básicas de saúde, como a inclusão de plantas medicinais (SOUZA et al., 2013). No Brasil, por sinal, os números são ainda maiores, pois um percentual de $91,9 \%$ da população, dizem já ter utilizado alguma planta medicinal, sendo que metade destes relatam cultivar essas plantas (ZAMBON et al., 2018).

No que diz respeito à capacitação e implementação das PIC em escala global, os percentuais são diversos, mas possuem grande representatividade, por exemplo com relação a profissionais de saúde que têm alguma formação em PIC perfazem, na Suíça (46\%), Inglaterra (50\%), França $(32 \%)$, sendo que a prescrição de plantas medicinais e ervas por médicos representam no Canadá (57\%), Alemanha (20\%), e Holanda (50\%) (TESSER et al., 2018).

Planta medicinal é conceituada como uma espécie vegetal, cultivada ou não, utilizada com propósitos terapêuticos e tem tradição de uso como remédio em uma população. Quando acaba de ser colhida, é classificada como planta fresca e, quando por processos de ressecamento e estabilização, planta seca. Quando a planta medicinal é industrializada para se obter um medicamento, tem-se como resultado o fitoterápico. O fitoterápico é produto obtido de matéria-prima ativa vegetal com finalidade profilática, curativa ou paliativa, podendo ser simples, quando o ativo é proveniente de uma única espécie vegetal medicinal, ou composto, quando o ativo é proveniente de mais de uma espécie vegetal (CRF-SP, 2019).

O tratamento terapêutico baseado na fitoterapia é realizado através de medicamento industrializado, manipulado ou ainda pelo uso da planta, por intermédio de preparações caseiras (RIBEIRO et al., 2018). As vantagens para sua aplicação são inúmeras: grande aceitação da população, inserção cultural da Fitoterapia nos usos e costumes da população, inclusão social (redução de desigualdades regionais por meio da geração local de renda), facilidade de acesso, a eficácia, menor potencial de provocar efeitos colaterais, a reprodutibilidade e constância da qualidade, ampliação das opções terapêuticas ofertadas aos usuários do Sistema Único de Saúde (SUS), desenvolvimento da cadeia produtiva e da indústria nacional assim como a excelente relação custo-benefício (VEIGA, 2008; HASENCLEVER et al., 2017; TESSER et al., 2018).

Acrescendo tais argumentos, os compostos naturais originados pelas espécies vegetais são divididos em dois grupos: os metabólitos primários, que atuam na síntese de compostos essenciais para a sobrevivência das espécies, cujas funções estão relacionadas aos aspectos estruturais e de armazenamento de energia, tais como, carboidratos, proteínas e lipídeos, enquanto que os metabólitos secundários, são originados a partir da conversão da energia luminosa em energia química (JAN et al., 2021). As plantas, em sua maioria, são ricas em compostos fenólicos, terpenóides e alcalóides. Ressalta-se que estes são sintetizados a partir dos metabólitos primários, sendo os responsáveis pelos efeitos medicinais, além de possuírem importância ecológica, em virtude de atuarem na atração de polinizadores, ou representar uma defesa química contra estresses ambientais (TUNGMUNNITHUM et al., 2018). Esses compostos são usados como medicamentos, aromas ou drogas relaxantes, especialmente óleos essenciais (SHARIFI-RAD et al., 2017). 
De acordo com a Agência Nacional de Vigilância Sanitária (ANVISA), existe uma infinidade de preparos para o uso das plantas medicinais, tais como a inalação, os gargarejos, os banhos de assento, a maceração, decocção, infusão (BRASIL, 2010). É importante lembrar, que um fitoterápico, ou seja, planta medicinal pode dar origem a uma droga vegetal, e ser comercializada sem processamento adicional, como por exemplo, na forma de um chá medicinal para uso em preparações através de decocção e infusão, etc., ou ainda essa droga vegetal (produto final), pode ser comercializada em outras formas farmacêuticas, como cápsulas, podendo conter excipientes. Já o derivado vegetal (produto final), pode ser obtido diretamente da planta medicinal ou da droga vegetal (BRASIL, 2014).

É de vital importância que os profissionais de saúde tenham conhecimento suficiente acerca da aplicação da fitoterapia, com o uso adequado desses compostos, haja vista que, apesar de serem substâncias naturalmente encontradas nas plantas pode oferecer riscos à saúde (FALZON; BALABANOVA, 2017). Outro ponto fundamental, a ser mencionado, é que algumas plantas medicinais já passaram por todas as etapas de pesquisa indispensáveis para atestar sua eficácia, sendo então permitidas para comporem o arsenal medicamentoso do profissional de saúde (MATTOS et al., 2018). Nesse sentido, é necessário a aplicação de normas para comprovação de segurança e eficácia de fitoterápicos, baseada em três critérios: por meio de estudos não clínicos e clínicos, por dados de literatura, por registro simplificado ou por credenciamento em virtude da tradicionalidade (BRASIL, 2014).

\section{OBESIDADE E CHÁ VERDE}

Vários agentes dietéticos que aumentam o gasto energético e diminuem 0 acúmulo de gordura, tem sido proposto como alternativa para combater a obesidade e suas comorbidades (BOLIN et al., 2020). No meio científico tem crescido o interesse pelo tratamento terapêutico contra a obesidade, baseado principalmente na fitoterapia como uma potencial alternativa, em virtude do baixo custo e poucos efeitos colaterais, tornando-os cada vez mais populares, já que são compostos extraídos de plantas medicinais, como a cafeína e as catequinas, presentes em chás e outros alimentos de origem vegetal (SCHWARTZ et al., 2017).

O consumo de chá verde tem mostrado alguns benefícios importantes para a saúde, já que possui propriedades funcionais e quando incluído na alimentação, pode trazer benefícios fisiológicos específicos. Além disso, os flavonóides, compostos bioativos do chá verde, possuem maior ação se somados a uma alimentação balanceada (ROBERTS et al., 2021).

Em vista disso, o chá verde é uma ferramenta bastante usual no controle da obesidade, consiste em uma bebida de sabor agradável, preparada por infusão de folhas frescas de Camellia Sinensis, rica em fitoquímicos, popular em vários países, devido não só ao seu sabor e aroma agradável, mas também aos efeitos benéficos para a saúde (SCHMITZ, 2005; SOUSA-FILHO et al., 2020). A produção ocorre através de um processo de vapor rápido para inibir a fermentação, causando a descoloração das folhas (BUDARAGA; PUTRA, 2020).

O consumo desse composto apresenta atividades biológicas positivas contra doenças crônicas como câncer, síndrome metabólica, diabetes mellitus do tipo 2 , patologias cardiovasculares (doenças cardíacas e cardiovasculares) e atua no antienvelhecimento (BOLIN et al., 2020). As catequinas presentes no chá verde 
podem impedir a ocorrência de câncer de pele, mama, próstata e pulmão, bem como, pode atuar na prevenção da deterioração dos nervos, como nas doenças de Alzheimer e Parkinson (BUDARAGA; PUTRA, 2020).

Os flavonóides representam o grupo de compostos fenólicos, categoria de compostos bioativos muito estudados, sendo subdivididos em: antocianinas, flavanas, flavononas, flavonas, flavonóis e isoflavonóides (JUCÁ et al., 2020). Possuem propriedades termogênicas devido à sua capacidade de agir em diferentes etapas de vias modulatórias como fosfodiesterase e catecol-O-metiltransferase (COMT) (BOLIN et al., 2020). Em pesquisas mais atuais, encontraram uma indução no fenótipo termogênico através da ativação de células beges em tecido adiposo branco e paralelamente redução no clareamento no tecido adiposo marrom (BOLIN et al., 2020).

Nota-se que dependendo do nível de fermentação, o chá oriundo das folhas da Camellia sinensis, é classificado em três tipos: chá verde, o qual não sofre fermentação durante o processamento, assim retém a cor original das folhas; o chá oolong o qual é parcialmente fermentado; e o chá preto, cujo processo de fermentação é maior, o que acarreta a coloração escurecida (SUN et al., 2018). O chá verde é uma folha jovem da planta Camellia sinensis, como dito anteriormente, é processada sem passar pela fermentação, possui composição química variada, que além das catequinas é composto de carboidrato, vitaminas (principalmente a vitamina $\mathrm{C}$ e K), sais minerais, metilxantinas, bem como, cafeína, a teofilina e a teobromina, as quais estimulam o Sistema Nervoso Central. Além disso, contém tanino e flúor (PRASANTH et al., 2019). É importante destacar que a presença de taninos e polifenóis do chá, se consumido em excesso, podem trazer prejuízos na biodisponibilidade de ferro, por esse motivo é interessante adotar medidas como, consumir o chá de duas a três após as refeições, aumentar o consumo de proteína e alimentos fonte de vitamina C (BUDARAGA; PUTRA, 2020).

Salienta-se ainda, que as folhas da erva Camellia Sinensis contêm mais de 2.000 componentes possuindo uma alta quantidade de polifenóis (cerca de 30-42\% do peso seco), sendo que, os compostos intitulados como catequinas representam $60-80 \%$ dessa quantidade (SHIXIAN et al., 2006). Nota-se que, os flavonóides e as catequinas possuem propriedades medicinais, como funções antioxidantes, quimioprotetora, termogênicas, antiinflamatória e anticarcinogênica (ROY et al., 2021).

Outrossim, o chá é considerado um antioxidante efetivo, já que é rico em flavonóis (300-400 mg/g). Protege o DNA contra lesões, pois cessa a iniciação e o progresso do crescimento de neoplasias estimulando ainda a apoptose (FILIPPINI et al., 2020). Além disso, atua contra doenças cardíacas, evitando a oxidação de LDL-C e inibindo formação de ateromas (FERNANDES et al., 2017).

As catequinas são potentes antioxidantes, sequestradores de radicais livres, quelantes de metais e inibidores de lipoperoxidação, sendo que as principais catequinas presentes no chá verde são: epicatequina (EC), epigalocatequina (EGC), epicatequina gallato (ECG) e epigalocatequina gallato (EGCG). As EGCG variam entre $2,31 \mathrm{mg}$ e $203,20 \mathrm{mg} / 100 \mathrm{~mL}$ de chá verde preparado por infusão, representando $50-80 \%$ do conteúdo total de catequina (ARRAES; FUNCHAL, 2017). Além disso, o chá é composto ainda por cafeína, teanina, teaflavinas, teorubiginas, quercetina e outros fenólicos, como ácido gálico e ácido clorogênico (BUDARAGA; PUTRA, 2020). 
Observa-se que a catequina EGCG atua na obesidade pois promove a redução de peso, já que diminui a diferenciação e proliferação dos adipócitos durante a lipogênese ( $\mathrm{CHO}$ et al., 2019). O chá verde possui um mecanismo de ação que atua no controle de peso, pois apresentando efeitos termogênicos, isso ocorre devido a uma interação de substâncias presentes no chá, como as catequinas (principalmente EGCG) e a cafeína (DULLOO et al., 1999), contudo, ainda não está claro se os benefícios para a saúde resultam do conteúdo de catequina, cafeína ou sinergismo entre os dois compostos (SOUSA-FILHO et al., 2020). Além disso, as interações da noradrenalina com as catequinas e a cafeína, promovem efeito termogênico, visto que a catequina inibe a enzima responsável por degradar a noradrenalina, a COMT, prolongando o efeito da noradrenalina na termogênese, aumentando a atividade do sistema nervoso simpático e estimulando a lipólise (HUANG et al., 2018). Já a cafeína (1 xícara de chá contém aproximadamente $6 \%$ de cafeína), provoca a inibição e degradação do AMP-C (que é uma resposta à noradrenalina). Essa concentração aumentada do AMP-C resultante da ação sinérgica entre cafeína e catequinas está relacionada ao efeito termogênico por estimular a produção de lipase hormônio sensível (LHS) (HUANG et al., 2018; RABIE; HO, 2019; TARIQUL ISLAM et al., 2019;). Dessa forma o consumo desse composto funcional atua na inibição da COMT e proporciona efeitos aumentados e prolongados na noradrenalina e aumenta o gasto energético e a oxidação de gordura em tecidos periféricos que contêm receptores adrenérgicos, como tecido adiposo (SOUSA-FILHO et al., 2020).

Em um estudo, o grupo que ingeriu maior quantidade de cafeína, dose acima do habitual (mais que $300 \mathrm{mg} / \mathrm{dia}$ ), demonstraram um resultado satisfatório na perda de peso e saciedade, apresentando menor quantidade de leptina, no entanto, o mesmo grupo não mostrou resultados positivos para manutenção do peso após o emagrecimento, isso pode ter ocorrido devido à perda de sensibilidade da cafeína (WESTERTERP-PLANTENGA, 2005).

Similarmente, a partir da avaliação do consumo de chá verde e sua relação com o emagrecimento em homens jovens e saudáveis, foi realizada a divisão dos participantes em três grupos: os que consumiram extrato de chá verde (contendo $90 \mathrm{mg}$ de EGCG e 50mg de cafeína), o outro grupo que consumiu apenas $50 \mathrm{mg}$ de cafeína isolada, e o terceiro grupo, que utilizou placebo constituído de celulose, constatando que o chá influenciou o gasto energético, com um aumento de $4 \%$, em relação à termogênese, concluindo assim que o polifenol mais abundante no chá verde, a EGCG, pode estimular a termogênese e a oxidação lipídica (DULLOO et al., 1999).

Do mesmo modo, em novo estudo, ratifica-se o efeito das catequinas sobre o peso corporal, demonstrando que essa substância é responsável pela redução dos níveis plasmáticos de colesterol total e triglicérides, bem como, pela diminuição do peso e da circunferência abdominal, estimulando a termogênese, promovendo maior gasto energético e ainda redução da ingesta alimentar, pela interação da EGCG com o receptor da leptina, aumentando a saciedade e proporcionando a perda de peso (HUANG et al., 2018; O'NEILL et al., 2018). Alguns estudos demonstram que os antioxidantes naturais, como os presentes na composição do chá verde, podem ser utilizados para atenuar níveis plasmáticos elevados de colesterol total, triglicérides e LDL - colesterol, bem como, atua no combate e na prevenção da obesidade (PRASANTH et al., 2019; GHOSH et al., 2021). 
O mecanismo de ação das catequinas no controle do peso se perfaz na regulação de várias enzimas que participam ativamente do processo de anabolismo e catabolismo lipídico do tecido adiposo, como acetilcoacarboxilase, ácido graxo sintetase, lipase pancreática e gástrica, sugerindo que ocorre a modulação da mitogênese, estimulação endócrina da função metabólica nas células de gordura (O’NEILL et al., 2018).

Sob o mesmo ponto de vista, foi avaliada a redução da gordura corporal em indivíduos eutróficos e com sobrepeso quando consumiram líquidos contendo catequinas. Esse estudo teve duração de 12 semanas, em que os indivíduos foram separados em três grupos, os quais receberam bebidas com diferentes concentrações de catequinas: a) grupo controle (41,1 mg/dia); b) grupo com baixa dose de ingestão (444,3 mg/dia); c) grupo com alta dose de ingestão (665,9 mg/dia). Os resultados do estudo revelaram um significativo decréscimo no peso corporal, no IMC, na circunferência da cintura e na relação cintura-quadril em ambos os grupos que ingeriram baixas e altas doses de catequinas, sendo que a medida da cintura indicou significativa redução na área total de gordura, bem como, na área de gordura visceral, em ambos os grupos (KAJIMOTO et al., 2005).

Trabalhos com extrato de chá verde evidenciaram que o uso crônico aumenta a oxidação de gorduras durante natação e corrida, além de auxiliar na perda de peso em ratos. Em humanos, uma pesquisa realizada com matcha $(4 \mathrm{~g}$ de pó de matcha sendo $3 \mathrm{~g}$ em um dia e $1 \mathrm{~g}$ no dia do teste, que representam $572 \mathrm{mg}$ de catequinas e 120mg de cafeína) em mulheres saudáveis identificou que 4 xícaras em 24 horas foi - suficiente para aumentar a oxidação de gorduras em exercícios de baixa intensidade, mas se mostrou indiferente em exercícios de moderada intensidade (WILLEMS et al., 2018). Em outro estudo realizado comparando o status antioxidante e metabólico do corpo em um grupo sob exercícios físicos intensos com ou sem extrato de chá verde demonstrou que, no grupo em que foram realizados treinos e ingestão do extrato concomitantemente, houve aumento de um marcador da obesidade, o SIRT1. Nesse caso, como a obesidade está relacionada à falta de SIRT1 sérica, o extrato mostrou-se capaz de aumentar esse marcador e melhorar o perfil antioxidante. Ele também é aumentado em estados catabólicos, demonstrando aumento do metabolismo das mulheres participantes do estudo (GHASEMI et al., 2020).

Por fim, alguns estudos, in vitro e em humanos, têm demonstrado que uma mistura de componentes do chá verde e cafeína aumentam a termogênese e a oxidação lipídica, promovendo gasto energético (GHOSH et al., 2021). Além disso, já foi demonstrado que as catequinas presentes no chá são capazes de reduzir peso corporal e gordura visceral e total (ROBERTS et al., 2021).

\section{CONSIDERAÇÕES FINAIS}

Com os dados apresentados neste trabalho, evidenciam que o chá verde, um alimento funcional, auxilia na prevenção e/ou tratamento da obesidade e suas comorbidades. Indubitavelmente, alimentos variados em base regular, avaliando os hábitos alimentares, a disponibilidade dos alimentos e as necessidades nutricionais do indivíduo são fundamentais e em dieta adequada. O chá verde parece ter efeitos positivos na prevenção e no tratamento da obesidade, principalmente por seu efeito termogênico, oriundo das catequinas cafeína presente em sua composição. Pesquisas relacionadas ao consumo ideal de chá verde sugerem que ele pode ter 
um efeito benéfico sobre a saúde humana, no entanto, os estudos ainda são inconclusivos quanto à dose diária recomendada. Sugere-se que mais estudos sejam realizados sobre a interação dos componentes do chá verde com os alimentos, a sua biodisponibilidade e os fatores anti nutricionais, assim como os potenciais efeitos adversos para que não acarretem problemas para grupos específicos como, por exemplo, mulheres grávidas, crianças e idosos.

\section{REFERÊNCIAS}

ALFERINK, L. J.; FITTIPALDI, J.; KIEFTE-DE JONG, J. C.; TAIMR, P.; HANSEN, B. E.; et al. Coffee and herbal tea consumption is associated with lower liver stiffness in the general population: the Rotterdam study. Journal of Hepatology, v. 67, n. 2, p. 339-348, 2017. DOI: https://doi.org/10.1016/j.jhep.2017.03.013

ARRAES, G. C. F.; FUNCHAL, C. Influência dos polifenois do chá verde na obesidade. Nutrição Brasil, v. 6, n. 1, p. 43-56, 2017.

BARAKAT, B.; ALMEIDA, M. E. F. Biochemical and immunological changes in obesity. Archives of Biochemistry and Biophysics, v. 708, 2021. DOI: https://doi.org/10.1016/j.abb.2021.108951

BOLIN, A. P.; SOUZA-FILHO, C. P. B.; DOS SANTOS, G. T. N.; FERREIRA, L. T.; DE ANDRADE, P. B. M.; et al. Adipogenic commitment induced by green tea polyphenols remodel adipocytes to a thermogenic phenotype. The Journal of Nutritional Biochemistry, v. 83 , p. 108429, 2020. DOI: https://doi.org/ 10.1016/j.jnutbio.2020.108429

BRASIL. Ministério da Saúde. Secretaria de Vigilância em Saúde. Departamento de Análise em Saúde e Vigilância de Doenças não Transmissíveis. Vigitel Brasil 2018: vigilância de fatores de risco e proteção para doenças crônicas por inquérito telefônico: estimativas sobre frequência e distribuição sociodemográfica de fatores de risco e proteção para doenças crônicas nas capitais dos estados brasileiros e no Distrito Federal em 2018. Brasília: Ministério da Saúde, 2019. Disponível em: https://portalarquivos2.saude.gov.br/images/pdf/2019/julho/25/vigitelbrasil-2018.pdf

BRASIL. Ministério da Saúde. Constituição da República Federativa do Brasil: texto constitucional promulgado em 5 de outubro de 1988, com as alterações determinadas pelas Emendas Constitucionais de Revisão nos 1 a 6/94, pelas Emendas Constitucionais nos 1/92 a 91/2016 e pelo Decreto Legislativo no 186/2008. Brasília: Senado Federal, Coordenação de Edições Técnicas, 2016.

BRASIL. Ministério da Saúde. Instrução Normativa n. 4 de 18 de junho de 2014. Determina a publicação do Guia de orientação para registro de Medicamento Fitoterápico e registro e notificação de Produto Tradicional Fitoterápico. Brasília - DF, seção 1, p. 13, 2014.

BRASIL. Ministério da Saúde. Portaria no. 971, de 3 de maio de 2006: aprova a Política Nacional de Práticas Integrativas e Complementares no SUS. 
Brasília/DF,

2006.

Disponível

em

http://www.crbm1.gov.br/Portaria\%20MS\%20971\%202006.pdf

BRASIL. Ministério da Saúde. Agência Nacional de Vigilância Sanitária. Resolução da Diretoria Colegiada n. 10, de 9 de março de 2010. Dispõe sobre a notificação de drogas vegetais junto à Agência Nacional de Vigilância Sanitária (ANVISA). Diário Oficial da União da República Federativa do Brasil, Brasília/DF, 2010.

Disponível

em:

<http://bvsms.saude.gov.br/bvs/saudelegis/anvisa/2010/res0010_09_03_2010.html>.

BUDARAGA, I. K.; PUTRA, D. P. Study of Green Tea Catechin Dipped with Moringa Leaves. IOP Conference Series: Earth and Environmental Science, v. 515, n. 1, p. 012027, 2020. DOI: https://doi.org/10.1088/1755-1315/515/1/012027

CECCHIN, B.; LOPES FERREIRA, R. Metodologias interdisciplinares para uma nova educação ambiental. Revista Meio Ambiente e Sustentabilidade, v. 10, n. 20, p. 30-45, 2021.

CHO, D.; JEONG, H. W.; KIM, J. K.; KIM, A. Y.; HONG, Y. D.; et al. Gallocatechin Gallate-Containing Fermented Green Tea Extract Ameliorates Obesity and Hypertriglyceridemia Through the Modulation of Lipid Metabolism in Adipocytes and Myocytes. Journal of Medicinal Food, v. 22, n. 8, p. 779-788, 2019. DOI: https://doi.org/10.1089/jmf.2018.4327

CRF-SP - CONSELHO REGIONAL DE FARMÁCIA DO ESTADO DE SÃO PAULO. Plantas Medicinais e Fitoterápicos. v. 4, p. 1-72, 2019.

DACAL, M. DEL P. O; SILVA, I. S. Impactos das práticas integrativas e complementares na saúde de pacientes crônicos. Saúde em Debate, v. 42, n. 118, p. 724-735, 2018. DOI: https://doi.org/10.1590/0103-1104201811815

DULLOO, A.; DURET, C.; ROHRER, D.; GIRARDIER, L.; MENSI, N.; et al. Efficacy of a green tea extract rich in catechin polyphenols and caffeine in increasing $24 \mathrm{~h}$ energy expenditure and fat oxidation in humans. American Journal Clinical Nutrition, v. 70, n. 6, p. 1040-1045, 1999. DOI: https://doi.org/10.1093/ajcn/ 70.6.1040.

FALZON, C. C.; BALABANOVA, A. Phytotherapy: An Introduction to Herbal Medicine. Primary Care: Clinics in Office Practice, v. 44, n. 2, p. 217-227, 2017. DOI: https://doi.org/10.1016/j.pop.2017.02.001

FERNANDES, D. Z; FAIX, P. N.; LEMKE, L.; PERUSSOLO, L.; WEBER, V.; et al. Efeitos do chá verde e do exercício físico sobre a composição corporal de pessoas obesas. Cinergis, v. 18, n. 2, p. 156-159, 2017. DOI: http://dx.doi.org/10.17058/cinergis.v18i2.8438

FILIPPINI, T.; MALAVOLTI, M.; BORRELLI, F.; IZZO, A. A.; FAIRWEATHER-TAIT, S. J.; et al. Green tea (Camellia sinensis) for the prevention of cancer. Cochrane 
Database Systematic Reviews, v. 3, n. 3, 2020. DOI: https://doi.org/10.1002/14651858.CD005004.pub3

GHASEMI, E.; AFZALPOUR, M. E.; NAYEBIFAR, S. Combined high-intensity interval training and green tea supplementation enhance metabolic and antioxidant status in response to acute exercise in overweight women. The journal of physiological sciences: JPS, v. 70, n. 1, p. 31, 2020. DOI: http://dx.doi.org/10.1186/s12576-02000756-z.

GHOSH, S.; MANCHALA, S.; RAGHUNATH, M.; SHARMA, G.; SINGH, A. K.; et al. Role of Phytomolecules in the Treatment of Obesity: Targets, Mechanisms and Limitations. Current Topics in Medicinal Chemistry, v. 21, n. 10, p. 863-877, 2021. DOI: https://doi.org/10.2174/1568026621666210305101804

GOULARTE, J.; SANTOS, N. Q.; ZIECH, A. R. D. Plantas medicinais: cultivo e conhecimento pela população urbana de Santa Helena/PR. Revista Brasileira Multidisciplinar. v. 24, n. 1, p. 89-102, 2021. DOI: https://doi.org/10.25061/25272675/ReBraM/2021.v24i1.932

HASENCLEVER, L.; PARANHOS, J.; COSTA, C. R.; CUNHA, G.; VIEIRA, D. A indústria de fitoterápicos brasileira: desafios e oportunidades. Ciência \& Saúde Coletiva, v. 22, n. 8 , p. 2559-2569, 2017. DOI: https://doi.org/10.1590/141381232017228.29422016

HUANG, L. H.; LIU, C.; WANG, L.; HUANG, C.; HSU, C. Effects of green tea extract on overweight and obese women with high levels of low density-lipoproteincholesterol (LDL-C): a randomised, double-blind, and cross-over placebo-controlled clinical trial. BMC complementary and alternative medicine, v. 18, n. 1, 2018. DOI: http://dx.doi.org/10.1186/s12906-018-2355-x

JAN, R.; ASAF, S.; NUMAN, M.; LUBNA; KIM, K.-M. Plant Secondary Metabolite Biosynthesis and Transcriptional Regulation in Response to Biotic and Abiotic Stress Conditions. Agronomy, v. 11, n. 5, 2021. DOI: https://doi.org/10.3390/agronomy11050968

JHEMES, I.; ARAÚJO, S.; NEGREIROS, P. D. S.; FRANÇA, A. R. S.; ROSA, G. D. S.; et al. A Diversidade da Flora Brasileira no Desenvolvimento de Recursos da Saúde. Revista Uningá Review, v. 31, n. 1, p. 35-39, 2017.

JUCÁ, M. M.; CYSNE FILHO, F. M. S.; DE ALMEIDA, J. C.; MESQUITA, D. D. S.; BARRIGA, J. R. M.; et al. Flavonoids: biological activities and therapeutic potential. Natural Product Research, v. 34, n. 5, p. 692-705, 2020. DOI: https://doi.org/10.1080/14786419.2018.1493588

KAJIMOTO, O.; KAJIMOTO, Y.; YABUNE, M.; NAKAMURA, T.; KOTANI K.; et al. Tea catechins with a galloyl moiety reduce body weight and fat. Journal Health Science, v. 51, n. 2, p. 161-71, 2005. 
MATTOS, G.; CAMARGO, A.; DE SOUZA, C.; ZENI, A. Plantas medicinais e fitoterápicos na Atenção Primária em Saúde: percepção dos profissionais. Ciência \& Saúde Coletiva, v. 23, n. 11, p. 3735-3744, 2018. DOI: https://doi.org/10.1590/1413812320182311.23572016

MIRANDA, K. V. L.; UHLMANN, L. A. C. Uso de fitoterápicos na atualidade: uma revisão de literatura. Pubsaúde, v. 6, p. 1-4, 2021. DOI: https://doi.org/10.31533/pubsaude6.a160

O'NEILL ROTHENBERG, D.; ZHOU, C.; ZHANG, L. A Review on the Weight-Loss Effects of Oxidized Tea Polyphenols. Molecules (Basel, Switzerland), v. 23, n. 5, 2018. DOI: http://dx.doi.org/10.3390/molecules23051176

OHISHI, T.; FUKUTOMI, R.; SHOJI, Y.; GOTO, S.; ISEMURA, M. The Beneficial Effects of Principal Polyphenols from Green Tea, Coffee, Wine, and Curry on Obesity. Molecules. v. 26, n. 2, 2021. DOI: https://doi.org/10.3390/molecules26020453

PEIXOTO, L.; MORIM, M. P. Coleções botânicas: documentação da biodiversidade brasileira. Ciência e Cultura, v. 55, n. 3, p. 21-24, 2003.

PELTZER, K.; PENGPID, S. The use of herbal medicines among chronic disease patients in Thailand: a cross-sectional survey. Journal of Multidisciplinary Healthcare, $\quad$ v. $22, \quad$ n. $12, \quad$ p. 573-582, $2019 . \quad$ DOI: https://doi.org/110.2147/JMDH.S212953

POSWAL, F. S.; RUSSELL, G.; MACKONOCHIE, M.; MACLENNAN, E.; ADUKWU, E. C.; et al. Herbal Teas and their Health Benefits: A Scoping Review. Plant Foods for Human Nutrition, v. 74, n. 3, p. 266-76, 2019. DOI: https://doi.org/10.1007/s11130-019-00750-w

PRASANTH, M. I.; SIVAMARUTHI, B. S.; CHAIYASUT, C.; TENCOMNAO, T. A Review of the Role of Green Tea (Camellia sinensis) in Antiphotoaging, Stress Resistance, Neuroprotection, and Autophagy. Nutrients. v. 11, n. 2, 2019. DOI: https://doi.org/10.3390/nu11020474

RABIE, B. M.; HO, J. K. The mechanism of action of Lipiburn on fat metabolism. Frontiers in bioscience (Landmark edition), v. 24, n. 3, p. 427-434, 2019. DOI: http://dx.doi.org/10.2741/4726

RIBEIRO, V. P.; ARRUDA, C.; ABD EL-SALAM, M.; BASTOS, J. K. Brazilian medicinal plants with corroborated anti-inflammatory activities: a review. Pharmaceutical Biology, v. 56, n. 1, p. 253-268, 2018. DOI: https://doi.org/10.1080/13880209.2018.1454480

ROBERTS, J. D.; WILLMOTT, A. G. B.; BEASLEY, L.; BOAL, M.; DAVIES, R.; et al. The Impact of Decaffeinated Green Tea Extract on Fat Oxidation, Body Composition and Cardio-Metabolic Health in Overweight, Recreationally Active Individuals. Nutrients, v. 13, n. 3, p. 764, 2021. DOI: https://doi.org/10.3390/nu13030764. 
ROY, P.; TOMASSONI, D.; TRAINI, E.; MARTINELLI, I.; MICIONI, D.I.; et al. Natural Antioxidant Application on Fat Accumulation: Preclinical Evidence. Antioxidants (Basel), v. 10, n. 6, 2021. DOI: https://doi.org/10.3390/antiox10060858

RUELA, L. O.; MOURA, C. C.; GRANDIM, C. V. C.; STEFANELLO, J.; IUNES, D. H.; et al. Implementação, acesso e uso das práticas integrativas e complementares no Sistema Único de Saúde: revisão da literatura. Ciência \& Saúde Coletiva, v. 24, n. 11, p. 4239-4250, 2019. DOI: https://doi.org/10.1590/1413-812320182411.06132018

SANTANA, M. D. O.; SÁ, J. S.; NEVES, A. F.; FIGUEREDO, P. G. J.; ARAÚJO, J. O poder das plantas medicinais: uma análise histórica e contemporânea sobre a fitoterapia na visão de idosas. Revista Multidebates, v. 2, n. 2, 2018.

SANTOS, V. P.; TRINDADE, L. M. P. A enfermagem no uso das plantas medicinais e da fitoterapia com ênfase na saúde pública. Revista Científica FacMais, v. 8, n. 1, 2017.

SARMA, S.; SOCKALINGAM, S.; DASH, S. Obesity as a multisystem disease: Trends in obesity rates and obesity-related complications. Diabetes, Obesity and Metabolism, Suppl 1, v. 3, n. 16, 2021. DOI: https://doi.org/10.1111/dom.14290

SCHMITZ, W.; SAITO, A. Y.; ESTEVÃO, D.; SARIDAKIS, H. O. O chá verde e suas ações como quimioprotetor. Semina: Ciências Biológicas e da Saúde, v. 26, n. 2, p. 119-130, 2005. DOI: https://doi.org/10.5433/1679-0367.2005v26n2p119

SCHWARTZ, M. W.; SEELEY, R. J.; ZELTSER, L. M.; DREWNOWSKI, A.; RAVUSSIN, E.; et al. Obesity Pathogenesis: An Endocrine Society Scientific Statement. Endocrine Reviews, n. 38, v. 4, p. 267-296, 2017. DOl: https://doi.org/10.1210/er.2017-00111

SHARIFI-RAD, J.; SUREDA, A.; TENORE, G. C.; DAGLIA, M.; SHARIFI-RAD, M.; et al. Biological Activities of Essential Oils: From Plant Chemoecology to Traditional Healing Systems. Molecules, v. 22, n. 1, 2017. DOI: https://doi.org/10.3390/molecules22010070

SHIXIAN, Q.; VANCREY, B.; SHI, J.; KAKUDA, Y.; JIANG, Y. Green tea extract thermogenesis-induced weight loss by epigallocatechin gallate inhibition of catecholO-methyltransferase. Journal of Medicinal Food, v. 9, n. 4, p. 451-8, 2006. DOI: https://doi.org/10.1089/jmf.2006.9.451

SOUSA-FILHO, C. P. B.; FARIA, H.; ESPOSITO, J.; MELO, A.; RIBEIRO, M.; et al. Green tea improves the metabolism of peripheral tissues in $\beta 3$-adrenergic receptorknockout mice. Pharmacological Research, v. 159, p. 104956, 2020. DOI: https://doi.org/10.1016/j.phrs.2020.104956

SOUZA, C. M.; BRANDÃO, D. O.; SILVA, M. S. P.; PALMEIRA, A. C.; SIMÕES, M. O. S.; et al. Utilização de Plantas Medicinais com Atividade Antimicrobiana por Usuários do Serviço Público de Saúde em Campina Grande - Paraíba. Revista Brasileira de 
Plantas Medicinais, v. 15, n. 2, p. 188-193, 2013. DOI: https://doi.org/10.1590/S1516-05722013000200004

SUN, H.; CHEN, Y.; CHENG, M.; ZHANG, X.; ZHENG, X.; et al. The modulatory effect of polyphenols from green tea, oolong tea and black tea on human intestinal microbiota in vitro. Journal of Food Science Technology, v. 55, n. 1, p. 399-407, 2018. DOI: https://doi.org/10.1007/s13197-017-2951-7

TARIQUL ISLAM, A. F. M.; SCAVELLO, M.; LOFTI, P.; DANIEL, D.; HALDEMAN, P.; et al. Caffeine inhibits PI3K and mTORC2 in Dictyostelium and differentially affects multiple other cAMP chemoattractant signaling effectors. Molecular and cellular biochemistry, v. 457, n. 1-2, 2019. DOI: 10.1007/s11010-019-03520-z

TESSER, C. D.; SOUSA, I. M. C. DE; NASCIMENTO, M. C. DO. Práticas Integrativas e Complementares na Atenção Primária à Saúde brasileira. Saúde em Debate, v. 42, n. spe1, p. 174-188, 2018. DOI: https://doi.org/10.1590/0103$11042018 \mathrm{~S} 112$

TUNGMUNNITHUM, D.; THONGBOONYOU, A.; PHOLBOON, A.; YANGSABAI, A. Flavonoids and Other Phenolic Compounds from Medicinal Plants for Pharmaceutical and Medical Aspects: An Overview. Medicines (Basel), v. 5, n. 3, 2018. DOI: https://doi.org/10.3390/medicines5030093

VEIGA, V. F. Estudo do consumo de plantas medicinais na Região Centro-Norte do Estado do Rio de Janeiro: aceitação pelos profissionais de saúde e modo de uso pela população. Revista Brasileira de Farmacognosia, v. 18, n. 2, p. 308-313, 2008. DOI: https://doi.org/10.1590/S0102-695X2008000200027

WESTERTERP-PLANTENGA, M. S.; LEJEUNE, M. P. G. M.; KOVACS, E. M. R. Body weight loss and weight maintenance in relation to habitual caffeine intake and green tea supplementation. Obesity research, v. 13, n. 7, p. 1195-1204, 2005. DOI: http://dx.doi.org/10.1038/oby.2005.142

WILLEMS, M. E. T.; ŞAHIN, M. A.; COOK, M. D. Matcha Green Tea Drinks Enhance Fat Oxidation During Brisk Walking in Females. International journal of sport nutrition and exercise metabolism, v. 28, n. 5, p. 536-541, 2018. DOI: http://dx.doi.org/10.1123/ijsnem.2017-0237

ZAMBON, C. P.; TIEGS, L. M. R.; CAMPANA, G. A.; NUNES, J. S. O uso de Medicamentos Fitoterápicos no Processo de Emagrecimento em Acadêmicos do Curso de Farmácia da Faculdade de Educação e Meio Ambiente - FAEMA. Revista Científica da Faculdade de Educação e Meio Ambiente, v. 9, p. 500-506, 2018. DOI: https://doi.org/10.31072/rcf.v9iedesp.621 